pour 29,4-96,8-18,8-106,8-47,0-85,2 calculées; (2) 304-73-7 pour 302,1-77,0-4,9; (3) 231-133-20 pour $230,6-133,9-19,5$; (4) $233-135-16$ pour $235,4-130,5-18,1$. On voit que dans tous les cas, ${ }^{1}$ la concordance est très remarquable. La race des trotleurs français est donc assimilable à une population panmictique pour tous les facteurs pris en considération dans cette étude.

\title{
UN EFFET APPARENT DU RANG DE NAISSANCE SUR I'INCIDENCE D'UN CARACTÈ丶RE QUALITATIF DANS L'ANALYSE DE DONNÉES FAMILIALES
}

J.-M. Goux. - Laboratoire de Génétique, École nationale supérieure agronomique, 35-Rennes.

Dans les études familiales de caractères qualitatifs, les données sont souvent obtenues par recensement des individus manifestant le caractère. On dispose donc d'une statistique des familles dont au moins un enfant est du type recherché. Par ce mode d'échantillonnage, il n'est pas possible de détecter toutes les familles dont les parents, sans manifester le caractère, sont capables de le transmettre. On n'atteindra pas celles dont aucun enfant ne présente le caractère sous le simple effet du hasard. Pour une dimension $n$ déterminée des tamilles, et si la probabilité $p$ d'apparition du caractère chez un enfant est la même dans toutes les familles étudiées, le nombre d'enfants qui le manifestent est distribué en loi binômiale tronquée. L'importance relative de la classe non-détectée varie en fonction inverse de la dimension de la famille. La probabilité de cette classe est $\mathrm{P}_{o}=(1-p)^{n}$ et si $n$ augmente, $\mathrm{P}^{o}$ diminue. En étudiant l'incidence du caractère en fonction du rang de naissance sur de telles données familiales, on constate que le biais ci-dessus, s'il n'est pas pris en considération, entraine un effet apparent du rang de naissance sur l'incidence du caractère. En effet, la contribution de chaque dimension de famille n'est pas la même aux divers rangs, ce qui entraîne une diminution de l'incidence du caractère lorsque le rang de naissance augmente. Cette diminution est évidemment dépourvue de signification biologique.

Nous avons observé un tel biais dans une statistique familiale relative à la luxation congénitale de la hanche dans une population humaine. Pour cette affection, la situation est encore compliquée du fait que la probabilité d'atteinte n'est pas la même dans les deux sexes, par suite d'une différence de pénétrance. Il faut alors faire appel à un modèle multinômial tronqué. La prise en compte du biais d'échantillonnage fait disparaitre l'effet apparent du rang.

\section{NON LINÉARITÉ DE LA RELATION ENTRE MORTALITÉ PÉRINATALE ET CONSANGUINITÉ DANS LA POPULATION FRANÇAISE}

\author{
F. REYNEs. - Laboratoire de Génétique, E.N.S.A., 35-Rennes.
}

L'objectif initial de la recherche était de répondre à la question : "les équilibres génétiques des populations humaines résultent-ils principalement des mécanismes mutationnel ou ségrégationnel? ». A travers des raisonnements très dispersés, on constate le recours à un même procédé de mesure basé sur l'effet de la consanguinité. Jusqu'à présent, les essais de mesure de l'effet de la consanguinité sur le "fardeau génétique " d'une population ont consisté à comparer un groupe de couples consanguins à l'ensemble de la population dont ils faisaient partie, en admettant en particulier l'hypothèse d'additivité des effets des divers loci portant des gènes défavorables. Par des techniques démographiques spécifiques, nous avons pu montrer que la mortalité périnatale somme de la mortinatalité et de la mortalité infantile endogène - n'est pas une fonction linéaire de la consanguinité : les hypothèses de base ne sont par conséquent pas valables, notamment celle concernant l'action indépendante des divers loci porteurs de gènes défavorables. Il n'est donc pas possible, sous ces hypothèses simplificatives, d'avoir une indication sur l'importance relative des 